Article - Discoveries

\title{
Pangenome evolution in environmentally transmitted symbionts of deep-sea mussels is governed by vertical inheritance
}

Devani Romero Picazo ${ }^{1}$, Almut Werner ${ }^{1}$, Tal Dagan $^{1}$, Anne Kupczok ${ }^{1,2,3^{*}}$

${ }^{1}$ Genomic Microbiology Group, Institute of General Microbiology, Christian-Albrechts University, Kiel, Germany

2 Max Planck Institute for Marine Microbiology, Bremen, Germany

${ }^{3}$ Bioinformatics Group, Wageningen University \& Research, Wageningen, the Netherlands

* corresponding author: anne.kupczok@wur.nl 
bioRxiv preprint doi: https://doi.org/10.1101/2021.11.12.468352; this version posted November 13, 2021. The copyright holder for this preprint (which was not certified by peer review) is the author/funder, who has granted bioRxiv a license to display the preprint in perpetuity. It is made available under aCC-BY-NC-ND 4.0 International license.

\begin{abstract}
Microbial pangenomes vary across species; their size and structure are determined by genetic diversity within the population and by gene loss and horizontal gene transfer (HGT). Many bacteria are associated with eukaryotic hosts where the host colonization dynamics may impact bacterial genome evolution. Host-associated lifestyle has been recognized as a barrier to HGT in parentally transmitted bacteria. However, pangenome evolution of environmentally acquired symbionts remains understudied, often due to limitations in symbiont cultivation. Using high-resolution metagenomics, here we study pangenome evolution of two co-occurring endosymbiont populations inhabiting individual Bathymodiolus brooksi mussels from a single cold seep. The symbionts, sulfur-oxidizing (SOX) and methane-oxidizing (MOX) gamma-proteobacteria, are environmentally acquired at an early developmental stage and individual mussels may harbor multiple strains of each species. We found differences in the accessory gene content of both symbionts across individual mussels, which are reflected by differences in symbiont strain composition. Compared to core genes, accessory genes are enriched in functions involved in genome integrity maintenance. We found no evidence for recent horizontal gene transfer between both symbionts. A comparison between the symbiont pangenomes revealed that the MOX population is less diverged and contains fewer accessory genes, supporting the view that the MOX association with $B$. brooksi is more recent than that of SOX. Our results show that the pangenomes of both symbionts evolved mainly by vertical inheritance. We conclude that association with individual hosts over their lifetime leads to genetically isolated symbiont subpopulations, constraining the frequency of HGT in the evolution of environmentally transmitted symbionts.
\end{abstract}

Key words: Pangenome, High-resolution metagenomics, horizontal gene transfer, accessory genome 


\section{Introduction}

Bacterial populations can show enormous genomic diversity, which comprises nucleotide differences between homologous sequences and variation in the accessory gene content. In particular, gene content diversity is described by the species pangenome, which consists of all the genomic sequences present across individuals of a bacterial species. The core genes in a pangenome are present in each individual while the remaining genes are considered as accessory (Brockhurst et al. 2019). Pangenome size and structure vary across bacterial species (Maistrenko et al. 2020) and pangenomic diversity is important for bacterial adaptation in environmental species, e.g., the marine Prochlorococcus (Kashtan et al. 2014). To understand microbial adaptation, it is thus crucial to understand the evolutionary processes that shape pangenome diversity. The main processes that give rise to microbial pangenomes are gene duplication and loss during vertical inheritance as well as gene acquisition via horizontal gene transfer (HGT). HGT enables the transfer of genetic material between microbial individuals that are not related by inheritance (Hall et al. 2017) and it is particularly relevant for the evolution of microbial pangenomes (Treangen and Rocha 2011; Tria and Martin 2021). Many mechanisms of HGT involve the activity of mobile genetic elements (MGEs) - such as phages, plasmids, or transposons - for transferring genetic material between different DNA strands.

Many bacterial species are known to lead a host-associated lifestyle, i.e., they are strictly or facultatively associated with eukaryotic hosts. Symbionts have different modes of transmission; parentally (or vertically) transmitted bacteria are transferred from adults to their progeny, while environmentally (or horizontally) transmitted bacteria are acquired from the environment either from a free-living population or other hosts. Mixed transmission modes are also common over long evolutionary time (Bright and Bulgheresi 2010; Russell 2019). The host association has important implications for the adaptation of symbionts via HGT since bacterial populations that share a habitat may be able to access the habitat-specific gene pool by HGT (Bordenstein and Reznikoff 2005; Newton and Bordenstein 2011; Polz et al. 2013). Indeed, several studies demonstrated that gene transfer from locally adapted populations may facilitate host colonization. For example, in plant-associated communities, MGEs enabled the adaptation of locally adapted nitrogen-fixing soil bacteria to associate with novel crops during their domestication (Greenlon et al. 2019) and in sponges, diverse functions that potentially provide a selective advantage to the symbionts in that niche were acquired by HGT (Robbins et al. 2021). Notably, these examples stem from environmentally transmitted symbionts that might have a wider potential for HGT compared to parentally transmitted symbionts. First, infection of a host by multiple symbionts results in a shared environment, where the chances for HGT are higher and second, genes can potentially be acquired from environmental bacteria during the free-living stage. Indeed, very few HGT events have been reported in well-studied insect symbioses, potentially due to genetic isolation linked to the intracellular lifestyle and parental transmission (PintoCarbó et al. 2016; López-Madrigal and Gil 2017; Waterworth et al. 2020).

Previous studies showed that the evolution of endosymbiont genomes is characterized by rare HGT and less accessory genes compared to environmental bacteria (Kloesges et al. 2011; Brockhurst et al. 2019). This conclusion was drawn based on a few model symbionts that have been cultivated and sequenced and that are mostly parentally transmitted. However, less is known about symbionts that are metabolically dependent on their host and hence cannot be well cultivated under laboratory conditions (Pande and Kost 2017) and were thus excluded from previous studies. In the last 20 years, cultivation-independent approaches, such as metagenomics, revolutionized our view of microbial diversity (e.g., (Giovannoni et al. 2014; Castelle and Banfield 2018)). Additionally, deeply sequenced metagenomes provide adequate datasets for studying variation within microbial species (Denef 2019; Rossum et al. 2020). Metagenomics approaches enable us to assess the variation of all organisms in a particular environment. This approach revealed abundant strain diversity in symbionts, e.g., in the gut microbiome of humans and bees (Ellegaard and Engel 2019; Garud et al. 2019). Additionally, the combination of metagenomics with pangenomes analysis provides links between strain occurrences and gene content variation (Delmont and Eren 2018; Utter et al. 2020). 
The presence of strain diversity has recently been reported for environmentally transmitted symbionts that reside in Bathymodiolus mussels. These symbionts are acquired during the mussels' metamorphosis from a planktonic to a benthic lifestyle and are hosted in bacteriocytes within the gill epithelium, where they provide the mussel with nutrition (Won et al. 2003; Franke et al. 2021). Bathymodiolus can be infected by two chemosynthetic symbiont species, sulfur-oxidizing (SOX) and methane-oxidizing (MOX) gamma-proteobacteria. Although most Bathymodiolus species harbor only a single $16 \mathrm{~S}$ phylotype for each symbiont, metagenomic analyses of multiple Bathymodiolus species showed that different SOX and MOX strains can be present within an individual mussel (Ansorge et al. 2019; Romero Picazo et al. 2019). An important role of MGEs and HGT in the evolution of SOX symbiont genomes from hydrothermal vents at the mid-atlantic ridge has been suggested. There, SOX genomes were found to contain high numbers of transposases, integrases, restriction-modification systems, and toxin-related genes, where the latter are also linked to MGEs (Sayavedra et al. 2015). In addition, it has been observed that co-occurring SOX strains from these sites differ in the content of genes involved in energy and nutrient utilization and viral defence mechanisms (Ansorge et al. 2019).

To study the evolution of pangenomes in symbiont populations, we here analyze the pangenomes of environmentally transmitted symbionts that reside in closely related, nearby hosts. To this end, 19 Bathymodiolus brooksi mussels were sampled from a single location at a cold seep site in the northern Gulf of Mexico and homogenized gill tissue of each mussel was deeply sequenced (Romero Picazo et al. 2019). Previous reconstruction of core-genome-wide strains revealed eleven SOX strains that group into four clades, and six MOX strains that group into two clades (Romero Picazo et al. 2019). Mussel individuals may harbor multiple strains of each species. The evolution of symbiont populations in individual mussels is characterized by genetic isolation, suggesting that symbionts are taken up at an early stage in the mussel life cycle and are then geographically isolated (Romero Picazo et al. 2019). Here, we study the effect of the geographical isolation on the SOX and MOX pangenome evolution. To this end, we analysed the population pangenomes of the SOX and MOX strains residing in these 19 mussels sampled from a single location.

\section{Results}

\section{A network approach for the recovery of symbiont pangenomes from microbiome metagenomes}

First we aimed to reconstruct the SOX and MOX pangenomes. To this end and in addition to the core genome, we recovered the accessory genome including the strain-specific genes. For that, we used a network approach, where the core genomes of both symbionts are used as a starting point for the expansion of the pangenome. Here, we identify connections between the symbiont genes, which are allocated in different metagenomic contigs in each sample. These connected contigs are therefore identified as symbiont-related (Table S1). Our pangenome inference revealed clear differences between the two symbionts. The pangenome of the SOX population comprises 2,484 genes with a total length of $2.27 \mathrm{Mbp}$. Of these, 962 (38.7\% of total genes) were identified as accessory, where the majority are single-copy accessory genes (Table 1). Each sample contains between 1,640 and 2,055 SOX genes (average 1,885) with genome lengths that range between 1.72 and $2.21 \mathrm{Mbp}$ (average 2.06 $\mathrm{Mbp}$ ) (Table S2A). The MOX population pangenome comprises 2,866 genes with a total length of 2.24 $\mathrm{Mbp}$, where 414 ( $14.5 \%$ of total genes) are accessory and the majority of accessory genes are singlecopy (Table 1). Each sample contains between 2,480 and 2,603 genes (average 2,546) with genome lengths between 2.39 and 2.50 Mbp (average 2.45 Mbp) (Table S2B). Both SOX and MOX core genomes have a relatively low $\mathrm{GC}$ content, where the $\mathrm{GC}$ content distributions over core genes are significantly different between SOX and MOX (Table 1, Fig. S1). In both symbionts, the GC content of the accessory genome is significantly lower than that of the core genome (Table 1, Fig. S1).

To evaluate the sensitivity of our approach to identify accessory genes, we estimated the recovery rate as the number of new genes that are added to the pangenome for each newly sampled mussel (Fig. S2A,B). We observed that $95 \%$ of the total accessory genome is detected when adding five samples for both bacterial species. This shows that our approach has the required sensitivity in order 
to recover accessory genes in the population pangenomes at this site. Sequencing coverage can further impact the sensitivity of our approach to recover pangenomes from metagenomic data. The two symbionts occur at different abundances within the mussel, which results in differences in the sequencing coverage of SOX and MOX. To investigate whether sequencing coverage might impact the sensitivity to detect accessory genes, we estimated the recovery rate for SOX when downsampling it to the MOX coverage. We found no decrease in the number of detected genes in SOX with this normalization, where also $95 \%$ of the total accessory genome is detected with five samples (Fig. S2C) and therefore the recovery of the accessory genome is comparable to the approach using the full coverage.

The pangenomes reconstructed here reflect the pangenomes of the population at the sampling site (i.e., not that of the entire species); nonetheless, the data enables us to compare the pangenome characteristics of the two symbionts. We found that the MOX population pangenome is larger than that of SOX, whereas the accessory genome is larger in SOX. The low GC content in both species is consistent with observations for other symbiotic bacteria (McCutcheon and Moran 2012). The lower GC content in the accessory genomes of both species may indicate 1 ) that genes are transferred from an external source with low GC content, or that genes with lower GC content are preferably transferred or 2) that accessory and core genes are under different selection regimes, where the higher GC content in the core genome is maintained by purifying selection (Bohlin et al. 2017).

The reconstruction of pangenomes from short-read metagenomic sequencing data is a challenging task. It is particularly difficult to infer unlinked mobile genetic elements such as plasmids from shortread metagenomes and furthermore to link them correctly to their bacterial hosts. This is thus not attempted here; instead, additional approaches such as isolation and sequencing, methylation patterns from long reads (Beaulaurier et al. 2018), or linking DNA by Hi-C (Yaffe and Relman 2020) would be necessary to resolve those mobile genetic elements with confidence and to link them to their host. Consequently, the inferred pangenomes reported here comprise the core and accessory genes located on the symbiont chromosomes.

\section{Gene content shows genetic isolation between mussels that is explained by strain composition.}

The intracellular lifestyle of the symbionts results in strong geographic isolation between mussels and this leads to genetically isolated symbiont populations (Romero Picazo et al. 2019). To study how genetic isolation impacts pangenome evolution in the mussel symbionts, we examined SOX and MOX gene content variation within and across individual mussels.

To analyze symbiont gene content diversity within individual mussels, we estimated the gene content diversity $\phi$ that is based on the relative frequency of genes in a population or subpopulation (see Methods). We find that $\phi$ is positively associated with the nucleotide diversity $\pi$ and both measures increase with $\alpha$-diversity estimated from the strain composition (Fig. 1A,B, S3A,B). Although $\pi$ is significantly lower in MOX than in SOX (two-sided Wilcoxon signed-rank test, $p$-value $=5.23 \mathrm{e}-4$ ), this is not observed for $\phi$ (two-sided Wilcoxon signed-rank test, $p$-value $=0.86$ ). The lower coverage of the MOX data could cause difficulties in estimating accurate gene frequencies and result in an overestimation of $\phi$. To investigate the impact of coverage on the $\phi$ estimation, we estimated $\phi$ for SOX downsampled to the MOX coverage. We find a strong correlation between SOX $\phi$ and $\phi$ for the downsampled data, where the downsampling results in a slight underestimation of $\phi$ (Fig. S3C). We therefore conclude that the high values of MOX $\phi$ cannot be explained by the lower MOX coverage.

To study the degree of isolation between mussels, we developed the pangenome fixation index $P_{S T}$, which is based on the gene content diversity $\phi$ and derived in analogy to the fixation Index $\left(F_{S T}\right)$ (see Methods). Small values of $F_{S T}$ or $P_{S T}$ indicate that the samples stem from the same population, whereas large values indicate that the samples constitute subpopulations. We found that $\mathrm{P}_{\mathrm{ST}}$ measures a lower degree of isolation than $\mathrm{F}_{S T}$, which is particularly pronounced in MOX (Fig. 1C,D, S4). This indicates that the degree of genetic isolation is stronger when measured at the level of single nucleotide variants (SNVs) in comparison to gene content. Despite $\mathrm{P}_{S T}$ being lower than $\mathrm{F}_{S T}$, both measures are correlated, 
where the correlation is especially strong for SOX. Furthermore, we observed that for both SOX and MOX, the pairwise correlation is highest when comparing $P_{S T}$ and $\beta$-diversity. Thus, the strain relationships and the strain distribution in the mussels - that are described by the $\beta$-diversity - are even stronger correlated to the pangenome fixation index than to the SNV-based fixation index. We observe that samples cluster based on these diversity measures, where the clustering based on $\mathrm{P}_{\mathrm{ST}}$ is very similar to the clustering based on $\beta$-diversity (Fig. S4). We observed before that MOX only clusters based on $\beta$-diversity, but not with $F_{S T}$ (Romero Picazo et al. 2019) (see also Fig. S4). Thus, the clustering of MOX samples can only be observed based on $\mathrm{P}_{\text {ST }}$ and $\beta$-diversity, but not based on $\mathrm{F}_{\text {ST. }}$.

Taken together, we derived methods to study population diversity and genetic isolation based on gene content. We find that genetic isolation based on gene content is highly associated with $\beta$-diversity based on strain composition. This supports that gene content variation is strongly associated with the strain relationships instead of being mobile between strains. Since $\beta$-diversity is mostly driven by differences among the strain clades rather than differences among strains within a clade, the strong correlation further suggests that gene content differences are mainly found among strain clades. We thus conclude that gene content differences between mussels are related to the strain compositions within the mussels and especially to their clade compositions.

\section{Strain clades are defined by gene content differences.}

In the previous section, we compared the gene content within and between individual mussels. Next, we aim to resolve the accessory gene content of individual strains by identifying the presence of genes in mussels infected by a single strain. We defined strains to be dominant in a mussel when their frequency in a sample is at least 0.7 . For a sample with a dominant strain, genes are present in a strain when their coverage is at least $50 \%$ of the median core genome coverage in that sample and we term all these genes to be "assigned to that strain" (Fig. S5). We further merged the strain-assigned genes of all samples with the same dominant strain. Similarly, clade-assigned genes resulted from merging the gene content of strains belonging to the same clade.

For both symbionts, the majority of the accessory genes could be assigned to strains (Fig. 2); we assigned 731 genes (76\% of the SOX accessory genes) to five SOX strains that are dominant in twelve mussel samples, where some genes were assigned to multiple strains (Fig. 2, Table S3A). The clades differ in the number of accessory genes, where clade $\mathrm{S} 2$ contains the largest number and clade $\mathrm{S} 1$ the smallest number of accessory genes. Additionally, S2 contains 169 genes that cannot be found in any other clade, i.e., they are clade-specific genes. In contrast, each of the other clades have at most 71 specific genes. We also identified 67 genes that were assigned to all strains, of which 18 are multicopy genes. For MOX, we could assign 276 accessory genes (67\% of the MOX accessory genes) to three different strains that were dominant in ten different samples (Fig. 2, Table S3B). Of these, 65 genes are specific to clade $M 1,132$ are specific to clade $M 2$, and 77 genes were shared among all three strains, of which 34 are multi-copy. We find that the reconstructed gene content of samples with the same dominant strain overlap to a large extent, which serves as additional support for the robustness of our approach (Table S3).

To gain insights into the pace of gene content evolution, we estimated pairwise strain distances in terms of differentially present genes and of SNVs. We found that strain pairs generally have more SNVs compared to differentially present genes (Fig. S6, Table S4), which supports that gene content changes occur less frequently than substitutions. The positive correlation between the number of differentially present genes and SNVs supports a positive relationship between gene content changes and substitutions, which is compatible with gene content evolution by vertical inheritance rather than frequent horizontal gene transfer.

Here, by extracting genes present in samples with a dominant strain, we could assign a large proportion of accessory genes to SOX and MOX strains. We find a high level of gene sharing among strains within the same clade, which supports that clades are characterized by gene content and that gene content evolves mainly by vertical inheritance. 


\section{The core genome is more diverged than the accessory genome for both symbionts.}

Considering our evidence that the symbiont pangenomes mainly evolve by vertical inheritance, we aimed to investigate the evolution of accessory gene sequences. To this end, we identified SNVs on all the genes in the pangenomes. The number of SNVs per kilo base pairs (SNVs/kbp) is higher in SOX than in MOX for all gene classes (core, single-copy accessory, and multi-copy accessory) (Table 1). For both species, the SNVs/kbp is lower in the accessory genome than in the core genome, where many of the single-copy accessory genes do not have any SNV (Fig. S7A, Table 1) - 475 genes in SOX (51\%) and 298 $(79 \%)$ in MOX. Furthermore, multi-copy genes have the highest SNVs/kbp (Table 1), which suggests that the divergence in multi-copy genes is overestimated due to the inclusion of paralogs.

To study the selection pressure on the pangenome, we estimated $\mathrm{pN} / \mathrm{pS}$ for the single-copy genes, where the analysis is restricted to genes with at least one SNV. In MOX, core and accessory genes show a similar pS distribution and also a similar pN/pS distribution (Fig. S7B,C). Thus, although divergence is low in MOX, the selection pressure acting on the core and accessory genome is similar. In contrast, we observed that the $\mathrm{pN} / \mathrm{pS}$ distribution is shifted to lower values for the SOX core genes compared to SOX accessory genes (Table 1, Fig. S7B). This could suggest that the strength of purifying selection is higher on the core genome. However, the pS distribution is shifted to higher values for the SOX core genes compared to the accessory genes (Table 1, Fig. S7C). It has been observed that the relative rate of nonsynonymous to synonymous substitutions depends on the divergence of the analyzed species (Rocha et al. 2006; Romero Picazo et al. 2019). Indeed, we find that the joint distribution of $\mathrm{pS}$ and $\mathrm{pN} / \mathrm{pS}$ are largely overlapping for the SOX core and accessory genome, with some accessory genes having a very low $\mathrm{pS}$ and a high $\mathrm{pN} / \mathrm{pS}$ (Fig. S8A). We thus cannot conclude that the strength of selection is different between SOX core and accessory genes.

To rule out the possible impact of differential coverage between core and accessory genes in the $\mathrm{pN}$ and $\mathrm{pS}$ estimates, we studied the distribution of these measures by only considering SNVs found across those samples containing a dominant strain. In these samples, SNV detection should be less biased by coverage because core and accessory genes have similar coverage (Fig. S5). Indeed, we find that the distributions of $\mathrm{pN} / \mathrm{pS}$ and $\mathrm{pS}$ estimated only from those samples are similar to the distributions previously estimated for the full dataset (Fig. S8), which suggests that variation in coverage does not explain the differences observed between the core and accessory genome.

The frequencies of SNVs/kbp presented here suggest that the accessory gene sequences are more conserved than the core genes in both symbionts. In a scenario where the accessory genes are enriched for mobile genes and have been taken up multiple times from the environmental gene pool, the accessory genome is expected to be very diverse. We thus conclude that this scenario is not realistic. In contrast, the majority of the accessory genome has probably been present in the ancestor of the population or has only been acquired once. Furthermore, the larger number of genes without detected mutations in the accessory genome of MOX compared to SOX suggests that gene family diversification is more recent in MOX compared to SOX. We could not detect a difference in selection pressure acting on the core and accessory genomes, mainly due to the low divergence level of these genes.

\section{Functions encoded by the accessory genome are mostly associated with the maintenance of genome integrity.}

To further investigate the origin of the accessory genes in the MOX and SOX symbiont populations, we studied the distribution of functional categories across the core and accessory genomes. Functional annotation of all genes in the pangenomes by clusters of orthologous groups (COGs) revealed that functions associated with central metabolism are overrepresented in the core genomes of both SOX and MOX pangenomes (COG categories "translation, ribosomal structure and biogenesis" and "aminoacid transport and metabolism"). In SOX, multiple additional categories related to central metabolism are overrepresented (Fig. 3A,B). 
The accessory genomes of both populations are overrepresented in functions associated with the maintenance of genome integrity, namely "Mobilome: prophages, transposons", "Defense mechanisms" and "DNA replication, recombination and repair". The COG category "Mobilome: prophages, transposons" is more prevalent in the MOX pangenome (62 genes in MOX, 26 genes in SOX). However, SOX shows a larger proportion of this category in the accessory genome $(19,73 \%)$ than $\operatorname{MOX}(17,27 \%)$. Investigating the functional annotation of the genes in detail, we identified 14 integrases in SOX and five in MOX. Additionally, we found 70 transposases in MOX and 20 in SOX. Of these, $16(23 \%)$ are identified as multi-copy genes in MOX, while three (15\%) are identified as multicopy genes in SOX.

The COG category "Defense mechanisms" is overrepresented in the accessory genomes of both populations ( 49 accessory out of 72 genes in SOX, 68\%; 23 accessory out of 54 genes in MOX, 43\%). We found a high number of genes associated with restriction-modification systems: 72 in MOX, where $24(33 \%)$ are accessory, and 81 in SOX, where 48 genes (59\%) are accessory. Additionally, we found a larger repertoire of CRISPR-Cas genes in SOX (20 genes, thereof 16 accessory) in comparison to MOX (seven genes, thereof none accessory). The prevalence of defense mechanisms opens the question, whether viruses are present in the mussel environment. The prediction of phages in all metagenomic contigs revealed 51 potential viral contigs, of which there is only one high-confidence complete phage contig (Table S5). Thus, viruses are either not highly abundant in the B. brooksi microbiome or could not be detected in the kind of data analyzed here.

Several functions are differentially present between the pangenomes of the symbionts (Fig. 3A,B). For example, we found that MOX contains 51 genes related to "Cell motility", where 43 (84\%) are in the core genome, whereas only three core genes are identified in SOX for this category. Additionally, 146 genes are assigned to "Signal transduction mechanisms" in MOX (21 accessory genes, 14\%), whereas only 31 genes are found in SOX (11 accessory genes, 35\%). To further examine the functions that are differentially present among strains, we inspected the genes that have been assigned to strains (Table S6). We observed that most of the strain-specific genes in SOX belong to the categories "Defense mechanisms" and "Replication, recombination and repair", followed by "Cell wall/membrane/envelope biogenesis" category. Notably the COG category "Cell wall/membrane/envelope biogenesis" also includes the toxin-related genes that have been described previously to be variable across strains (Sayavedra et al. 2015). In MOX, the categories "Defense mechanisms", "Replication, recombination and repair", and "Cell wall/membrane/envelope biogenesis" also differ between strains and, additionally, MOX strains differ in genes involved in "Signal Transduction mechanisms". For SOX and MOX, categories related to metabolism are rarely found to be strain-specific. The differences among the strain-specific functional categories suggest that the different strains differ in the repertoire of genes that are involved in interactions between organisms, including interactions with the mussel host and with other bacteria or mobile elements.

We conclude that in both symbiont pangenomes, the accessory genomes comprise mostly genes that function in genome integrity maintenance. The high prevalence of transposases and restrictionmodification systems suggests that genome rearrangements contribute to gene content variation among strains. Additionally, we find that MOX has a larger repertoire of mobilome-related genes and contains more genes related to cell motility and signal transduction.

\section{HGT within the mussel environment plays a minor role in symbiont evolution.}

For endosymbiotic bacteria, the concept of an "intracellular arena" posits that the host cell serves as an arena for bacteria, where symbionts can acquire putatively beneficial genes from a niche-specific gene pool (Bordenstein and Reznikoff 2005; Newton and Bordenstein 2011). To identify mobile elements with the potential to transfer genes in that environment, we studied the diversity of transposases in the pangenomes. Transposases can duplicate within or between genomes, where recent duplications will show a low diversity between both transposase copies. Here, we observed a low genetic diversity only among transposases within the same species, whereas the minimum 
divergence between species is 0.50 substitutions per site (Fig. S9). Thus, recent transposase duplication events have occurred only within species and not between species, which leads to the conclusion that there is no evidence for recent transfer of transposases between both symbiont species.

In addition, we aimed to identify possible HGT events between SOX and MOX symbionts. To this end, we inferred 760 homologous protein pairs between the two symbionts. Of these, 709 (93\%) comprise core genes from both species, where the majority encode for central metabolism functions (Fig. $3 \mathrm{C}$ ). The homologs that are core genes of both species are presumably related by common ancestry, i.e., they are orthologs. In addition, 51 homologs involve at least one accessory gene (Table S7, Fig. 3C). These are candidates for a recent horizontal transfer between symbionts, where a transfer would result in a high sequence similarity between the homologs. However, the sequence identity between homologous pairs does not reach high values (less than 86\%, Fig. 4). In addition, the distributions of sequence identity of core pairs and of pairs involving at least one accessory gene are not significantly different (Wilcoxon rank sum test p-value= 0.3502; Fig. 4). We conclude that the homologous pairs involving an accessory gene are indeed orthologs, which were differentially lost in one or several strains, rather than being acquired recently by HGT between the symbionts.

Note that our approach might not be able to detect very recent transfers between SOX and MOX where both homologs are present in all of the samples. This limitation exists because a very recent between-species transfer within a single mussel may lead to discontinuous assemblies and exclusion of the transferred gene from both SOX and MOX contigs. However, transferred genes that accumulated mutations in the donor or recipient should be distinguishable during the assembly. Our approach is expected to perform well in detecting HGT events, where homologs are differentially present in the different mussels or where homologs accumulated more than $5 \%$ nucleotide divergence. That said, we do not find any gene cluster (i.e., a group of genes with less than $5 \%$ nucleotide divergence) that is present in both pangenomes and we only find homologs between SOX and MOX with less than $86 \%$ amino acid identity, which are presumably orthologs. Consequently, we conclude that HGT between the symbiont species is rare.

\section{Discussion}

Here we used metagenomes to analyze the gene content of two co-occurring symbiont species that inhabit mussels from a single geographical site. We reconstructed the population pangenomes of the sampling sites by applying a novel approach that links accessory genes to core genes across the chromosomal contigs of multiple samples. Our reconstruction of the site pangenome revealed commonalities and differences in the evolution of the two symbionts. Whereas the SOX pangenome is smaller than that of MOX, the former has a higher fraction of accessory genes. The large SOX accessory genome is consistent with the recent finding that gene content variation among coexisting thiotrophic bacteria is common (Ansorge et al. 2020). We find that functions associated with the maintenance of genome integrity are consistently present in the accessory genomes of both species. Among them are genes encoding for defense mechanisms, in particular, genes related to restrictionmodification systems. In addition to defense, restriction-modification systems can also function as mobile genetic elements. They have, for example, been shown to be involved in genome rearrangements in termite gut symbionts and in gene birth and death in the human gut bacterium Helicobacter pylori (Furuta et al. 2011; Zheng et al. 2016).

We find that mobile genetic elements, such as transposons, are more prevalent in MOX, which also has a high proportion of them in the core genome. Furthermore, MOX has a higher fraction of genes related to cell motility and signal transduction, which can be found in the core and accessory genome. Notably, both these functional categories have been found to be underrepresented in intracellular compared to free-living bacteria (Merhej et al. 2009; Lo et al. 2016); thus, they are more relevant for a free-living lifestyle. This suggests that MOX still pursues an active free-living life stage. Additionally, the association of MOX with Bathymodiolus might be recent and ancient genes can still be found in 
the genome. The latter is consistent with the low level of divergence observed in MOX, both in terms of gene content differences and in terms of nucleotide diversity. A low level of diversity is expected for a young population or for a population of low effective size that was subject to recent bottleneck events (e.g., as in founder effects). Mussel phylogenies indeed support that the association of Bathymodiolus with MOX is younger than that with SOX. The clade comprising B. brooksi evolved from an ancestor with only the SOX symbiont about 10 million years ago (Lorion et al. 2013). Nevertheless, co-speciation of hosts and symbionts is rare in that system (Won et al. 2008) and it can thus not be ruled out that SOX and MOX symbiont populations have been replaced multiple times over mussel evolution.

Differences in pangenome size can also be caused by different rates of HGT. Nevertheless, we conclude that HGT is rare for both species. This conclusion is supported by the observations (i) that gene content differences between mussel individuals reflect the differences in strain composition, (ii) that the accessory genome is less diverged than the core genome for both symbionts, and (iii) that the homologs between SOX and MOX are not highly similar. Observations (i) and (ii) support that accessory genes have been acquired at most once from the environmental gene pool and then evolved by descent with modification within the symbiont lineages. Gene loss or recombination within the strains might also contribute to accessory gene evolution, whereas multiple transfer from the environmental pool or horizontal gene transfer between different strain clades is rare. Since additionally the reconstruction of homologs between SOX and MOX also did not reveal signals of recent gene transfer between the species (observation iii), we conclude that also HGT between the two species is rare. This contrasts their common ecology and their potential ability to access the habitat-specific gene pool as described for other species (Bordenstein and Reznikoff 2005; Newton and Bordenstein 2011; Polz et al. 2013). Notably, in SOX from different Bathymodiolus host species, a higher fraction of genes that potentially originated by HGT has been previously found (Sayavedra et al. 2015). Considering that our analysis is restricted to events within the SOX population at a particular site, this discrepancy may stem from differences in the sampling distribution of the compared strains. Furthermore, hostsymbiont interactions can differ on the molecular level between different Bathymodiolus species and their symbionts (Ponnudurai et al. 2017; Ponnudurai et al. 2020), which might also result in differences in the evolution between symbionts of different Bathymodiolus species.

Notably, our conclusion that HGT is rare for SOX and MOX contrasts what is known for most other bacterial species, where HGT is a major evolutionary driver (Treangen and Rocha 2011; Brockhurst et al. 2019). The absence of evidence for HGT in this environment might be explained by the absence of DNA transfer mechanisms in the symbionts or by environmental properties. Regarding the latter, the intracellular environment may interfere with mechanisms that rely on the transfer of free DNA such as natural transformation, since the DNA could be quickly degraded by the prevalent mussel digestive enzymes (Ponnudurai et al. 2017). Likely HGT mechanisms in such an environment are such where the DNA is transferred in a packaged manner (such as in phages, gene transfer agents, or outer membrane vesicles) or transferred in direct contact between donor and recipient (as in conjugation). However, these mechanisms are most likely to transfer genes between symbionts within a single bacteriocyte and only a few symbionts are generally harboured within a single bacteriocyte (Dubilier et al. 1998). We thus conclude that potential gene transfer events rarely establish in the population and that the host association results in genetically isolated subpopulations where HGT is limited. This is consistent with the observation that endosymbionts are rarely connected in gene transfer networks (Popa et al. 2011). Parentally transmitted symbionts are also known to have low rates of HGT (McCutcheon and Moran 2012; Latorre and Manzano-Marín 2017). Thus, the host association leads to genetically isolated subpopulations and, by limiting horizontal gene transfer, it impacts the pangenome evolution for symbionts of different transmission modes, including environmental, parentally, and also mixed modes of transmission.

We conclude that the low frequency of gene transfer within the mussel environment limits the recombination within the species. Together with the observation that symbiont populations within 
individual mussels are geographically isolated (Romero Picazo et al. 2019), we conclude that evolution of strain clades is governed by vertical inheritance. In the absence of HGT, gene content evolution is mainly driven by differential gene loss. Additionally, the high prevalence of mobile genes in the accessory genomes of the symbionts suggests that genome rearrangements play a role in the intraspecies gene content evolution. Genetic isolation and limited access to recombination are known drivers of genome degradation in parentally transmitted endosymbionts (McCutcheon and Moran 2012; Latorre and Manzano-Marín 2017). In contrast, in environmentally transmitted symbionts, even low rates of recombination can rescue the genomes from extensive degradation (Russell et al. 2020). Given that we do not observe highly reduced genomes, we thus conclude that occasional genetic recombination also contributes to the evolution of the SOX and MOX populations described here. Taken together, our analysis demonstrates the importance of vertical inheritance in contrast to horizontal gene transfer to the evolution of symbiont population pangenomes.

\section{Materials and Methods}

\section{Collection, sequencing, and core genome strain reconstruction}

Details of the sample processing and sequencing can be found in (Romero Picazo et al. 2019). In brief, 23 Bathymodiolus brooksi mussels were collected from a cold seep location at the northern Gulf of Mexico and mussel gill homogenate has been sequenced using Illumina HiSeq2500 (raw reads BioProject PRJNA508280). Individual samples were assembled with metaSPAdes (Nurk et al. 2017) and predicted genes were clustered with identity of $95 \%$ into gene clusters that represent the nonredundant gene catalog. Next, we performed co-abundance gene segregation by using a canopy clustering algorithm (Nielsen et al. 2014), which groups gene clusters into bins that covary in their abundances across the different samples. Two metagenomic species (MGS) were classified as the SOX and MOX core genome, respectively, and lowly and highly covered genes were further filtered. We additionally identified a third MGS (MGS3), which is present in a single sample in low abundance and for which no taxonomy could be inferred. After discarding samples with high variance in symbiont marker gene coverages and with low coverage after binning, 19 mussel samples remained as the basis for the following analysis. Single nucleotide variants (SNVs) were detected on the non-redundant gene catalog as described in (Romero Picazo et al. 2019). Strain reconstruction for the core genomes has been performed with DESMAN (Quince et al. 2017). In addition to the analysis presented in (Romero Picazo et al. 2019), singletons that originated from discarded samples were removed from the core genomes. This results in a SOX core genome of 1,408 genes and a MOX core genome of 2,443 genes.

\section{Pangenome reconstruction}

Differences in strain composition generate different assembly fragmentation patterns across samples, where regions of the genome that are present only in specific strains tend to result in isolated contigs. Here, we restore the linkage between contigs that belong to the same species and identify, from all the genes present in the catalog, the accessory and multi-copy genes that belong to SOX and MOX symbiont pangenomes. Our approach is based on the non-redundant gene catalog, which contains clusters of highly similar genes (sharing at least $95 \%$ of sequence identity) that are identified as gene clusters present across samples.

We use a network traversal approach, where the genes that are part of core gene clusters are used as initial seeds. First, we locate the seed genes on the contigs of the 19 samples. The first layer of the pangenome contains all additional gene clusters having gene members that are found on any of those contigs. Next, we use the genes that are part of these newly added gene clusters as seeds to expand the network. The extension of the network continues in an iterative manner until no additional genes can be added to the pangenome. Microbial genomes contain multi-copy genes that can be transferred at a relatively high rate within and between genomes, e.g., transposons. The use of multi-copy genes as seeds would potentially cause the spurious linkage of genome fragments. In order to avoid such artifacts, we only consider those genes as seeds that originate from clusters with sequence identity of at least 0.95 and that contain at most one gene from each sample. Nevertheless, as multi-copy genes 
can naturally be found in bacterial genomes, they were included in the pangenomes. We discarded a singleton gene from the SOX core genome connecting to the MOX pangenome. The link between the two pangenomes occurs in the second layer of the network, when two contigs containing each two genes in each pangenome were connected by another contig containing two genes. Although the SOX singleton certainly has coverage corresponding to a core gene, it only is present in one sample and has no functional annotation. Therefore, we suspect that the singleton has been misassigned as a SOX core gene and decided to not include it in the analysis. To avoid misassignment of genes in the MOX pangenome, we additionally discarded the two genes belonging to the contig connecting both pangenomes as well.

The quality of the pangenomes has been assessed by studying the distribution of two different gene cluster features across the pangenome layers, the cluster size and the cluster sequence identity (Table S1). For clusters added to layers of the pangenome, the gene cluster size is below 19 (the number of samples). This is expected for gene clusters that are not present in every strain and supports their status as accessory. Additionally, the median sequence identity of the clusters is close to one $(>0.99)$, which supports that the gene clusters added to the pangenomes are not affected by contamination i.e., genes from a different MGS.

We denote the additional genes added to the network as "accessory" genes, where genes with higher coverage than a core gene in any sample are denoted as "multi-copy accessory", in contrast to "singlecopy accessory". In addition to the core genes, we observed clusters of genes showing a similar coverage to the median core genome coverage across samples (median sample coverage $\pm 0.15 \mathrm{x}$ median core sample coverage for all samples, 114 SOX genes, 9 MOX genes). These genes are added to the core genome for all the presented analyses.

The metagenome-assembled genomes (MAGs) of both symbionts retrieved across all different samples have been deposited on the NCBI database under the Bioproject ID PRJNA508280 with BioSample IDs SAMN21876924-SAMN21876961. The protein sequences for both symbiont pangenomes have been deposited in the GitHub repository https://github.com/deropi/BathyBrooksiSymbionts.

\section{Measures of population diversity and diversification}

SNV data is used for calculating intra-sample and inter-sample nucleotide diversity $(\pi)$ and $\mathrm{F}_{\mathrm{ST}}$ and strain frequency and relatedness is used for calculating $\alpha$ - and $\beta$-diversity as described previously (Romero Picazo et al. 2019).

To estimate the degree of genetic isolation based on gene content, we have derived the intra- and inter-sample gene diversity measures. Analogous to the nucleotide diversity $\pi$ which estimates the average frequency of nucleotide differences over all pairs in a sample, we here define the gene diversity $\varphi$ which estimates the average frequency of gene content differences. For frequencies estimated from metagenomes, it is estimated as: $\phi(S)=\frac{2}{|G|} \sum_{i=1}^{|G|}\left(\frac{\gamma_{i, S} \min \left(0, C_{S}-\gamma_{i, S}\right)}{C_{S}\left(C_{S}-1\right)}\right)$, where $G$ is the number of genes in the pangenome, $\gamma_{i, S}$ is the coverage of gene $i$ in sample $S$ (measured as mean coverage across gene positions) and $C_{s}$ is the median coverage of core genes in sample $S$. Note that the minimum ensures that the difference between accessory gene coverage and median core coverage cannot be negative. Note that this definition differs from the previously defined measure of genome fluidity (Kislyuk et al. 2011).

The inter-sample gene diversity is then estimated as: $\phi\left(S_{1}, S_{2}\right)=\frac{1}{|G|} \sum_{i=1}^{|G|}\left(\frac{\gamma_{i, S_{1}} \min \left(0, C_{S_{2}}-\gamma_{i, S_{2}}\right)}{C_{i, S_{1}} C_{i, S_{2}}}\right)+$ $\left(\frac{\gamma_{i, S_{2}} \min \left(0, C_{S_{1}}-\gamma_{i, S_{1}}\right)}{C_{i, S_{1}} C_{i, S_{2}}}\right)$, where $S_{1}$ and $S_{2}$ correspond to the two samples compared. Finally, analogous to $F_{S T}$, we define the pangenome fixation index $P_{S T}$ which measures the genetic differentiation based on the gene diversity present within and between populations: $P_{S T}\left(S_{1}, S_{2}\right)=1-\frac{\phi\left(S_{1}\right)+\phi\left(S_{2}\right)}{2 \phi\left(S_{1}, S_{2}\right)}$. 
$\mathrm{pN} / \mathrm{pS}$, a variant of $\mathrm{dN} / \mathrm{dS}$ that can be used based on intra-species SNVs, is estimated as described previously (Romero Picazo et al. 2019). pN/pS was estimated individually for each of the genes in the two symbiont species as well as for the core and accessory genome for each symbiont.

The scripts to calculate these statistics have been deposited at https://github.com/deropi/BathyBrooksiSymbionts.

\section{Reconstruction of strain gene content}

To assign the accessory genes to particular strains, we first identified samples with a dominant strain, where a dominant strain is defined as having a frequency of at least 0.7. In these samples, accessory genes with a coverage of at least $50 \%$ of the median core coverage are assigned to the dominant strain. Strain-assigned genomes were reconstructed by merging all the genes assigned to the same strain across samples. Therefore, the clade-assigned genomes were inferred by merging all the genes assigned to strains of a particular clade.

\section{Functional annotation and overrepresentation analysis}

Functional annotation by Clusters of Orthologous Groups (COGs) was determined by eggNOG-mapper v2 (Huerta-Cepas et al. 2019). Genes with multiple COGs contribute to the counts of each of the COGs. The overrepresentation of gene categories in either core or accessory genomes was done by performing multiple Fisher's exact tests for each COG category with FDR p-value correction.

Transposase genes were identified as genes containing the substring 'Transposase' or 'transposase' and integrase genes were identified as genes containing the substring 'Integrase' or 'integrase'. Additionally, we used HMMer (Eddy 2011; Eddy 2019) to screen for functional domains with hmmsearch (e-value <1e-4) against Pfam (Mistry et al. 2021) that are related to restrictionmodification and CRISPR-Cas. The list of Pfam accessions used is provided in Table S8, where restriction-modification profiles have been taken from (Croucher et al. 2014) and CRISPR-Cas profiles were directly extracted by looking for the keyword 'CRISPR-Cas' in the Pfam portal (http://pfam.xfam.org/search).

\section{Phage identification}

All contigs of the 19 samples were screened for viral sequences in two steps. First, contigs with VirSorter (Roux et al. 2015) categories 1, 2, and 4 were retained. Second, genes on those contigs were searched with hmmscan (e-value <1e-5) (Eddy 2011; Eddy 2019) against pVOGs (Grazziotin et al. 2017) and contigs were considered as viral, if they have at least three pVOGs, where at least two pVOGs have a viral quotient of at least 0.8 . Phage contigs were clustered with public phages using vConTACT2 (Jang et al. 2019). Average amino acid identity (AAI) has been calculated with CompareM (Parks 2020).

\section{Transposase genetic distance estimation}

To estimate the degree of divergence among transposases in the population, pairwise alignments between all pairs of transposases were reconstructed with MAFFT auto mode (Katoh and Standley 2013) and pairwise distances are estimated with the $K 80$ model implemented in the $R$ package ape (Paradis and Schliep 2019).

\section{Homologous proteins between SOX and MOX}

To identify homologous proteins, we extracted reciprocal best blast (Altschul et al. 1990) hits between the translated genes in the SOX and MOX pangenomes. Then, full-length protein sequence alignments were reconstructed with the Needleman-Wunsch algorithm implemented in EMBOSS (Needleman and Wunsch 1970; Rice et al. 2000), and pairs of genes with pairwise identities of at least $30 \%$ were assigned as homologous. 


\section{Acknowledgements}

We like to thank Nicole Dubilier for the collection of the original data and valuable discussions on the manuscript. Additionally, we thank Marina Khachatutyan for her comments on the manuscript. This work was supported by the CRC1182 Origin and Function of Metaorganisms.

\section{References}

Altschul SF, Gish W, Miller W, Myers EW, Lipman DJ. 1990. Basic local alignment search tool. J. Mol. Biol. 215:403-410.

Ansorge R, Romano S, Sayavedra L, Porras MÁG, Kupczok A, Tegetmeyer HE, Dubilier N, Petersen J. 2019. Functional diversity enables multiple symbiont strains to coexist in deep-sea mussels. Nat. Microbiol. 4:2487-2497.

Ansorge R, Romano S, Sayavedra L, Rubin-Blum M, Gruber-Vodicka H, Scilipoti S, Molari M, Dubilier N, Petersen J. 2020. The hidden pangenome: comparative genomics reveals pervasive diversity in symbiotic and free-living sulfur-oxidizing bacteria. Available from: https://www.biorxiv.org/content/10.1101/2020.12.11.421487v1

Beaulaurier J, Zhu S, Deikus G, Mogno I, Zhang X-S, Davis-Richardson A, Canepa R, Triplett EW, Faith $\mathrm{JJ}$, Sebra R, et al. 2018. Metagenomic binning and association of plasmids with bacterial host genomes using DNA methylation. Nat. Biotechnol. 36:61-69.

Bohlin J, Eldholm V, Pettersson JHO, Brynildsrud O, Snipen L. 2017. The nucleotide composition of microbial genomes indicates differential patterns of selection on core and accessory genomes. BMC Genomics 18:151.

Bordenstein SR, Reznikoff WS. 2005. Mobile DNA in obligate intracellular bacteria. Nat. Rev. Microbiol. 3:688-699.

Bright M, Bulgheresi S. 2010. A complex journey: transmission of microbial symbionts. Nat. Rev. Microbiol. 8:218-230.

Brockhurst MA, Harrison E, Hall JPJ, Richards T, McNally A, MacLean C. 2019. The Ecology and Evolution of Pangenomes. Curr. Biol. 29:R1094-R1103.

Castelle CJ, Banfield JF. 2018. Major New Microbial Groups Expand Diversity and Alter our Understanding of the Tree of Life. Cell 172:1181-1197.

Croucher NJ, Coupland PG, Stevenson AE, Callendrello A, Bentley SD, Hanage WP. 2014. Diversification of bacterial genome content through distinct mechanisms over different timescales. Nat. Commun. 5:5471.

Delmont TO, Eren AM. 2018. Linking pangenomes and metagenomes: the Prochlorococcus metapangenome. PeerJ 6:e4320.

Denef VJ. 2019. Peering into the Genetic Makeup of Natural Microbial Populations Using Metagenomics. In: Polz MF, Rajora OP, editors. Population Genomics: Microorganisms. Population Genomics. Cham: Springer International Publishing. p. 49-75. Available from: https://doi.org/10.1007/13836_2018_14

Dubilier N, Windoffer R, Giere O. 1998. Ultrastructure and stable carbon isotope composition of the hydrothermal vent mussels Bathymodiolus brevior and B. sp. affinis brevior from the North Fiji Basin, western Pacific. Mar. Ecol. Prog. Ser. 165:187-193.

Eddy SR. 2011. Accelerated Profile HMM Searches. PLOS Comput. Biol. 7:e1002195.

Eddy SR. 2019. HMMER 3.3. Available from: http://hmmer.org/.

Ellegaard KM, Engel P. 2019. Genomic diversity landscape of the honey bee gut microbiota. Nat. Commun. 10:446.

Franke M, Geier B, Hammel JU, Dubilier N, Leisch N. 2021. Coming together-symbiont acquisition and early development in deep-sea bathymodioline mussels. Proc. R. Soc. B Biol. Sci. 288:20211044.

Furuta Y, Kawai M, Yahara K, Takahashi N, Handa N, Tsuru T, Oshima K, Yoshida M, Azuma T, Hattori $\mathrm{M}$, et al. 2011. Birth and death of genes linked to chromosomal inversion. Proc. Natl. Acad. Sci. U. S. A. 108:1501-1506. 
Garud NR, Good BH, Hallatschek O, Pollard KS. 2019. Evolutionary dynamics of bacteria in the gut microbiome within and across hosts. PLOS Biol. 17:e3000102.

Giovannoni SJ, Cameron Thrash J, Temperton B. 2014. Implications of streamlining theory for microbial ecology. ISME J. 8:1553-1565.

Grazziotin AL, Koonin EV, Kristensen DM. 2017. Prokaryotic Virus Orthologous Groups (pVOGs): a resource for comparative genomics and protein family annotation. Nucleic Acids Res. 45:D491-D498.

Greenlon A, Chang PL, Damtew ZM, Muleta A, Carrasquilla-Garcia N, Kim D, Nguyen HP, Suryawanshi V, Krieg CP, Yadav SK, et al. 2019. Global-level population genomics reveals differential effects of geography and phylogeny on horizontal gene transfer in soil bacteria. Proc. Natl. Acad. Sci. 116:15200-15209.

Hall JPJ, Brockhurst MA, Harrison E. 2017. Sampling the mobile gene pool: innovation via horizontal gene transfer in bacteria. Phil Trans R Soc B 372:20160424.

Jang HB, Bolduc B, Zablocki O, Kuhn JH, Roux S, Adriaenssens EM, Brister JR, Kropinski AM, Krupovic $M$, Lavigne $R$, et al. 2019. Taxonomic assignment of uncultivated prokaryotic virus genomes is enabled by gene-sharing networks. Nat. Biotechnol. 37:632-639.

Kashtan N, Roggensack SE, Rodrigue S, Thompson JW, Biller SJ, Coe A, Ding H, Marttinen P, Malmstrom RR, Stocker R, et al. 2014. Single-cell genomics reveals hundreds of coexisting subpopulations in wild Prochlorococcus. Science 344:416-420.

Katoh K, Standley DM. 2013. MAFFT multiple sequence alignment software version 7: improvements in performance and usability. Mol. Biol. Evol. 30:772-780.

Kislyuk AO, Haegeman B, Bergman NH, Weitz JS. 2011. Genomic fluidity: an integrative view of gene diversity within microbial populations. BMC Genomics 12:32.

Kloesges T, Popa O, Martin W, Dagan T. 2011. Networks of gene sharing among 329 proteobacterial genomes reveal differences in lateral gene transfer frequency at different phylogenetic depths. Mol. Biol. Evol. 28:1057-1074.

Latorre A, Manzano-Marín A. 2017. Dissecting genome reduction and trait loss in insect endosymbionts. Ann. N. Y. Acad. Sci. 1389:52-75.

Lo W-S, Huang Y-Y, Kuo C-H. 2016. Winding paths to simplicity: genome evolution in facultative insect symbionts. FEMS Microbiol. Rev. 40:855-874.

López-Madrigal S, Gil R. 2017. Et tu, Brute? Not Even Intracellular Mutualistic Symbionts Escape Horizontal Gene Transfer. Genes 8:247.

Lorion J, Kiel S, Faure B, Kawato M, Ho SYW, Marshall B, Tsuchida S, Miyazaki J-I, Fujiwara Y. 2013. Adaptive radiation of chemosymbiotic deep-sea mussels. Proc $R$ Soc $B$ 280:20131243.

Maistrenko OM, Mende DR, Luetge M, Hildebrand F, Schmidt TSB, Li SS, Rodrigues JFM, Mering C von, Coelho LP, Huerta-Cepas J, et al. 2020. Disentangling the impact of environmental and phylogenetic constraints on prokaryotic within-species diversity. ISME J.:1-13.

McCutcheon JP, Moran NA. 2012. Extreme genome reduction in symbiotic bacteria. Nat. Rev. Microbiol. 10:13-26.

Merhej V, Royer-Carenzi M, Pontarotti P, Raoult D. 2009. Massive comparative genomic analysis reveals convergent evolution of specialized bacteria. Biol. Direct 4:13.

Minh BQ, Schmidt HA, Chernomor O, Schrempf D, Woodhams MD, von Haeseler A, Lanfear R. 2020. IQ-TREE 2: New Models and Efficient Methods for Phylogenetic Inference in the Genomic Era. Mol. Biol. Evol. 37:1530-1534.

Mistry J, Chuguransky S, Williams L, Qureshi M, Salazar GA, Sonnhammer ELL, Tosatto SCE, Paladin L, Raj S, Richardson L, et al. 2021. Pfam: The protein families database in 2021. Nucleic Acids Res. 49:D412-D419.

Needleman SB, Wunsch CD. 1970. A general method applicable to the search for similarities in the amino acid sequence of two proteins. J. Mol. Biol. 48:443-453.

Newton ILG, Bordenstein SR. 2011. Correlations between bacterial ecology and mobile DNA. Curr. Microbiol. 62:198-208. 
Nielsen HB, Almeida M, Juncker AS, Rasmussen S, Li J, Sunagawa S, Plichta DR, Gautier L, Pedersen AG, Le Chatelier E, et al. 2014. Identification and assembly of genomes and genetic elements in complex metagenomic samples without using reference genomes. Nat. Biotechnol. 32:822-828.

Nurk S, Meleshko D, Korobeynikov A, Pevzner PA. 2017. metaSPAdes: a new versatile metagenomic assembler. Genome Res. 27:824-834.

Pande S, Kost C. 2017. Bacterial Unculturability and the Formation of Intercellular Metabolic Networks. Trends Microbiol. 25:349-361.

Paradis E, Schliep K. 2019. ape 5.0: an environment for modern phylogenetics and evolutionary analyses in R. Bioinformatics 35:526-528.

Parks D. 2020. dparks1134/CompareM. Available from: https://github.com/dparks1134/CompareM

Pinto-Carbó M, Sieber S, Dessein S, Wicker T, Verstraete B, Gademann K, Eberl L, Carlier A. 2016. Evidence of horizontal gene transfer between obligate leaf nodule symbionts. ISME J. 10:2092-2105.

Polz MF, Alm EJ, Hanage WP. 2013. Horizontal gene transfer and the evolution of bacterial and archaeal population structure. Trends Genet. TIG 29:170-175.

Ponnudurai R, Heiden SE, Sayavedra L, Hinzke T, Kleiner M, Hentschker C, Felbeck H, Sievert SM, Schlüter R, Becher D, et al. 2020. Comparative proteomics of related symbiotic mussel species reveals high variability of host-symbiont interactions. ISME J. 14:649-656.

Ponnudurai R, Kleiner M, Sayavedra L, Petersen JM, Moche M, Otto A, Becher D, Takeuchi T, Satoh $\mathrm{N}$, Dubilier N, et al. 2017. Metabolic and physiological interdependencies in the Bathymodiolus azoricus symbiosis. ISME J. 11:463-477.

Popa O, Hazkani-Covo E, Landan G, Martin W, Dagan T. 2011. Directed networks reveal genomic barriers and DNA repair bypasses to lateral gene transfer among prokaryotes. Genome Res 21:599-609.

Quince C, Delmont TO, Raguideau S, Alneberg J, Darling AE, Collins G, Eren AM. 2017. DESMAN: a new tool for de novo extraction of strains from metagenomes. Genome Biol. 18:181.

Rice P, Longden I, Bleasby A. 2000. EMBOSS : The European Molecular Biology Open Software Suite. Trends Genet. 16:276-277.

Robbins SJ, Song W, Engelberts JP, Glasl B, Slaby BM, Boyd J, Marangon E, Botté ES, Laffy P, Thomas $\mathrm{T}$, et al. 2021. A genomic view of the microbiome of coral reef demosponges. ISME J. 15:1641-1654.

Rocha EPC, Smith JM, Hurst LD, Holden MTG, Cooper JE, Smith NH, Feil EJ. 2006. Comparisons of $\mathrm{dN} / \mathrm{dS}$ are time dependent for closely related bacterial genomes. J. Theor. Biol. 239:226-235.

Romero Picazo D, Dagan T, Ansorge R, Petersen JM, Dubilier N, Kupczok A. 2019. Horizontally transmitted symbiont populations in deep-sea mussels are genetically isolated. ISME J. 13:2954-2968.

Rossum TV, Ferretti P, Maistrenko OM, Bork P. 2020. Diversity within species: interpreting strains in microbiomes. Nat. Rev. Microbiol. 18:491-506.

Roux S, Enault F, Hurwitz BL, Sullivan MB. 2015. VirSorter: mining viral signal from microbial genomic data. PeerJ 3:e985.

Russell SL. 2019. Transmission mode is associated with environment type and taxa across bacteriaeukaryote symbioses: a systematic review and meta-analysis. FEMS Microbiol. Lett. 366:fnz013.

Russell SL, Pepper-Tunick E, Svedberg J, Byrne A, Castillo JR, Vollmers C, Beinart RA, Corbett-Detig R. 2020. Horizontal transmission and recombination maintain forever young bacterial symbiont genomes. PLOS Genet. 16:e1008935.

Sayavedra L, Kleiner M, Ponnudurai R, Wetzel S, Pelletier E, Barbe V, Satoh N, Shoguchi E, Fink D, Breusing $C$, et al. 2015. Abundant toxin-related genes in the genomes of beneficial symbionts from deep-sea hydrothermal vent mussels. elife 4:e07966.

Treangen TJ, Rocha EPC. 2011. Horizontal transfer, not duplication, drives the expansion of protein 
families in prokaryotes. PLoS Genet. 7:e1001284.

Tria FDK, Martin WF. 2021. Gene Duplications Are At Least 50 Times Less Frequent than Gene Transfers in Prokaryotic Genomes. Genome Biol. Evol. [Internet] 13. Available from: http://academic-oup-com/gbe/article/13/10/evab224/6380140

Utter DR, Borisy GG, Eren AM, Cavanaugh CM, Mark Welch JL. 2020. Metapangenomics of the oral microbiome provides insights into habitat adaptation and cultivar diversity. Genome Biol. 21:293.

Waterworth SC, Flórez LV, Rees ER, Hertweck C, Kaltenpoth M, Kwan JC. 2020. Horizontal Gene Transfer to a Defensive Symbiont with a Reduced Genome in a Multipartite Beetle Microbiome. mBio 11:e02430-19.

Won Y-J, Hallam SJ, O'Mullan GD, Pan IL, Buck KR, Vrijenhoek RC. 2003. Environmental Acquisition of Thiotrophic Endosymbionts by Deep-Sea Mussels of the Genus Bathymodiolus. Appl. Environ. Microbiol. 69:6785-6792.

Won Y-J, Jones WJ, Vrijenhoek RC. 2008. Absence of Cospeciation Between Deep-Sea Mytilids and Their Thiotrophic Endosymbionts. J. Shellfish Res. 27:129-138.

Yaffe E, Relman DA. 2020. Tracking microbial evolution in the human gut using Hi-C reveals extensive horizontal gene transfer, persistence and adaptation. Nat. Microbiol. 5:343-353.

Zheng H, Dietrich C, Hongoh Y, Brune A. 2016. Restriction-Modification Systems as Mobile Genetic Elements in the Evolution of an Intracellular Symbiont. Mol. Biol. Evol. 33:721-725. 


\section{Figure legends}
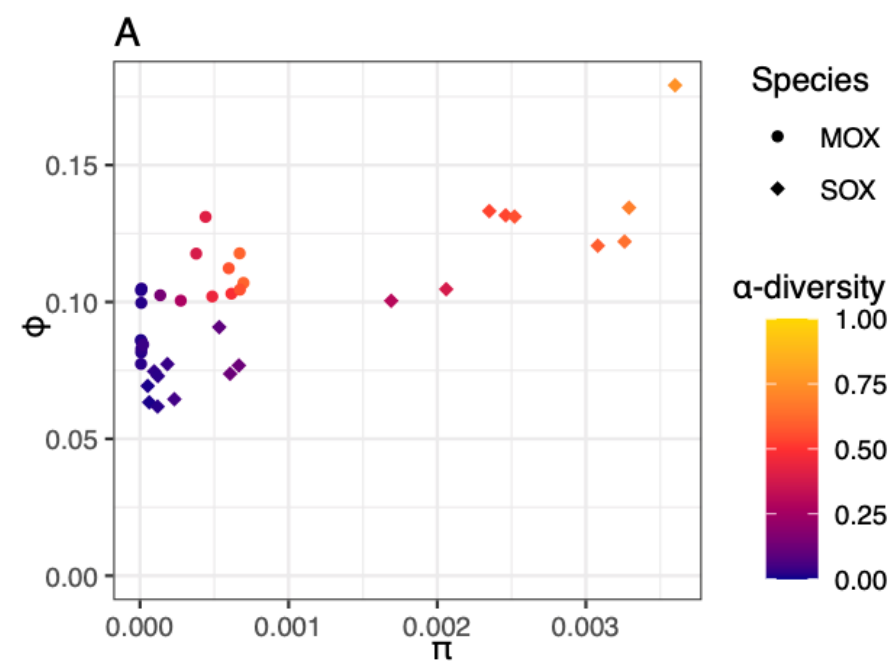

B
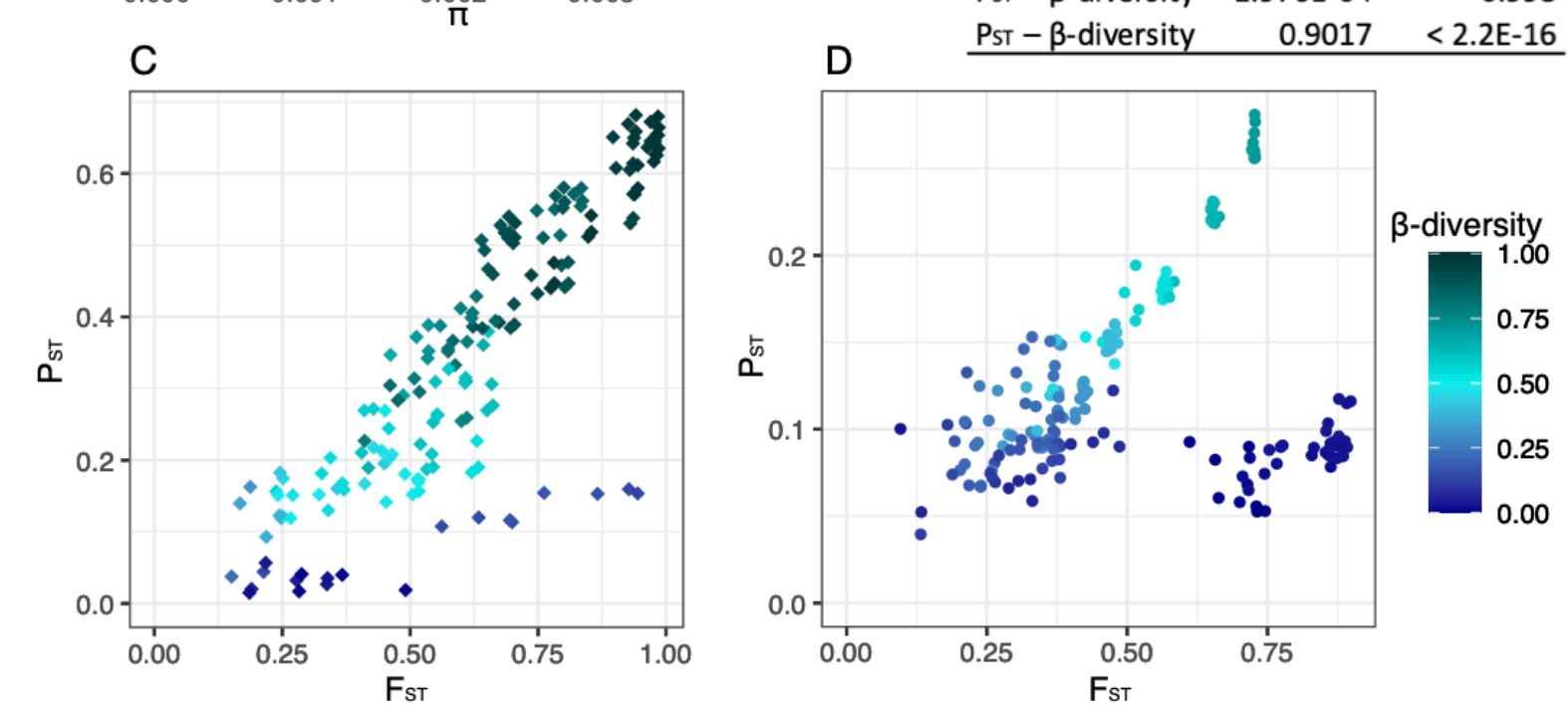

Figure 1. Relationships between different measures for population diversity and genetic isolation. A) Relationships between nucleotide diversity $(\pi)$, gene content diversity $(\phi)$, and $\alpha$-diversity for SOX and MOX. B) Correlation coefficients and $p$-values for Pearson's product-moment correlation. C) Relationships between fixation index $\left(F_{S T}\right)$, pangenome fixation index $\left(P_{S T}\right)$, and $\beta$-diversity for SOX. $P_{S T}$ (median 0.3417 ) is significantly lower than $\mathrm{F}_{\mathrm{ST}}$ (median 0.6216) (two-sided Wilcoxon signed-rank test, $\mathrm{p}$-value $<2.2 \mathrm{e}-16)$. D) Relationships between fixation index $\left(\mathrm{F}_{S T}\right)$, pangenome fixation index $\left(\mathrm{P}_{S T}\right)$, and $\beta$-diversity for MOX. PST (median 0.1043) is significantly lower than $\mathrm{F}_{S T}$ (median 0.4290) (two-sided Wilcoxon signed-rank test, $\mathrm{p}$-value $<2.2 \mathrm{e}-16$ ). 

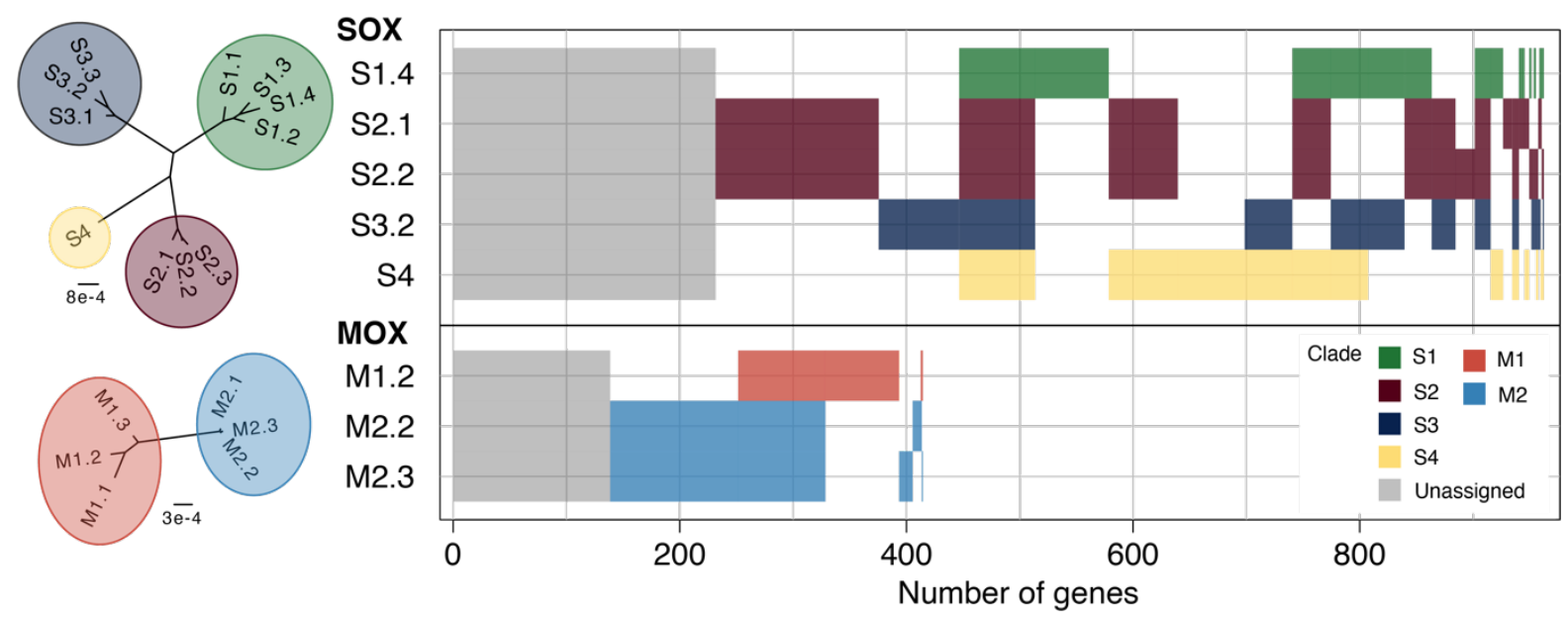

Figure 2. Presence-absence patterns for reconstructed strains in SOX and MOX. Gray genes are accessory genes that could not be assigned to any strain, whereas colors indicate the clade affiliation. For interpretation, core strain phylogenies are shown on the left. Based on the concatenated core gene alignment, IQ-TREE 2 using the GTR+G8 model and 1000 approximate bootstrap replicates resolved these trees with all branches supported $>85 \%$ (Minh et al. 2020). 
A

SOX

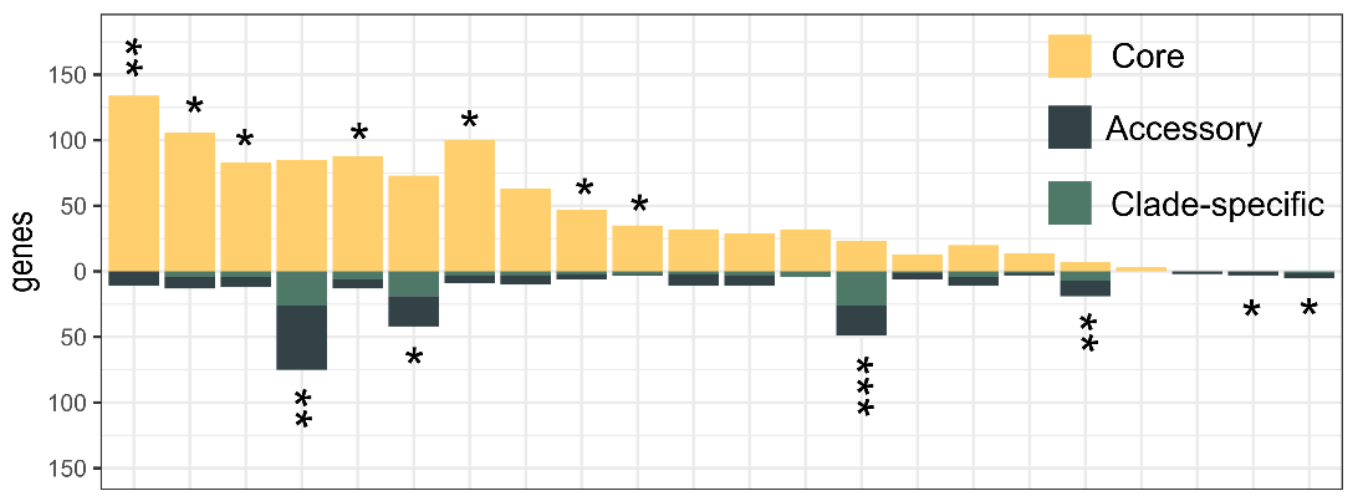

B

MOX

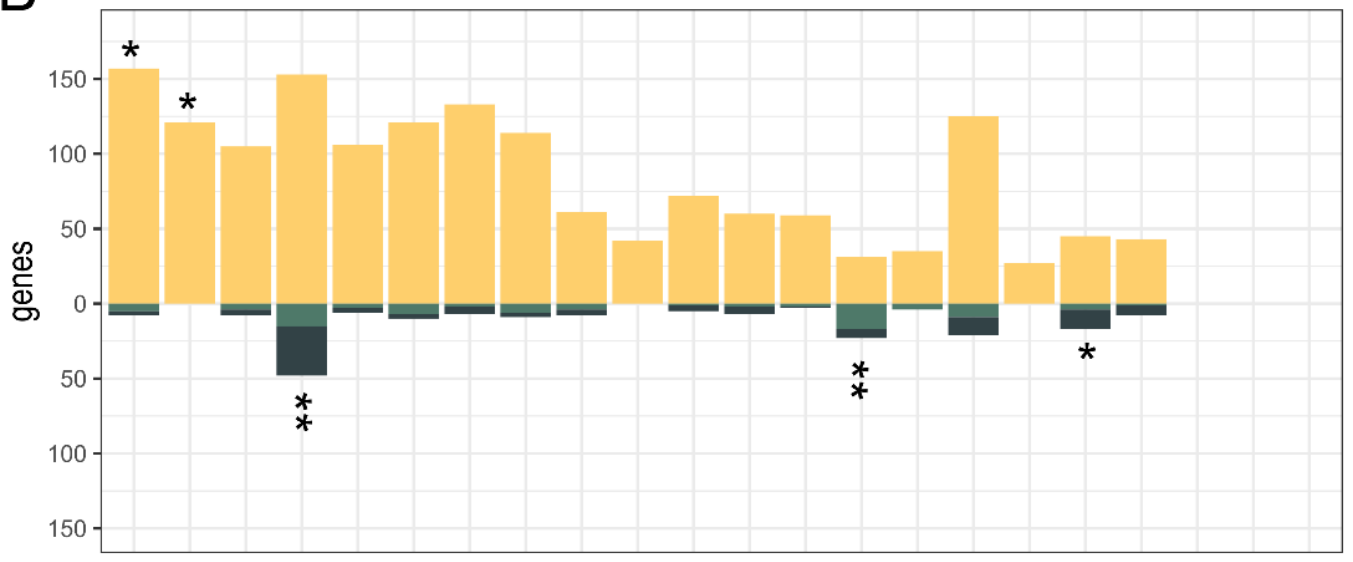

C

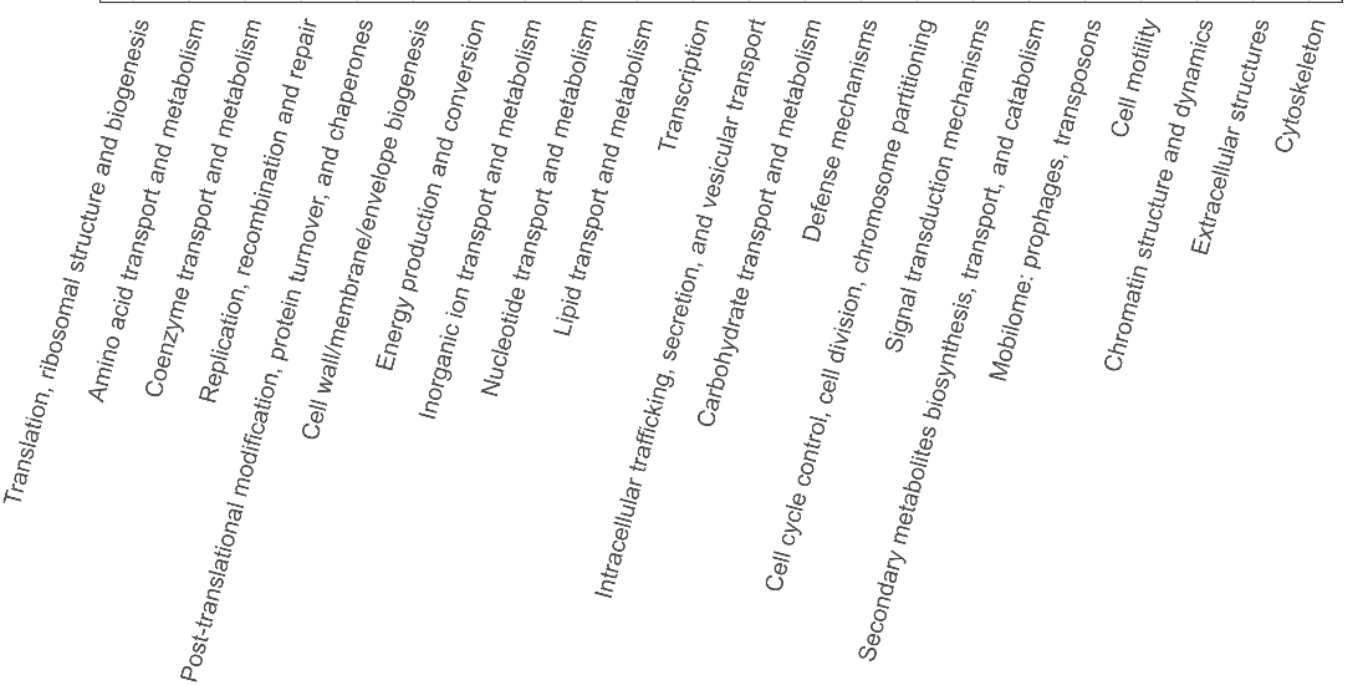


bioRxiv preprint doi: https://doi.org/10.1101/2021.11.12.468352; this version posted November $13,2021$. The copyright holder for this preprint (which was not certified by peer review) is the author/funder, who has granted bioRxiv a license to display the preprint in perpetuity. It is made available under aCC-BY-NC-ND 4.0 International license.

Figure 3. COG annotations for SOX and MOX pangenomes. Distribution of COG annotations for A) SOX and B) MOX core and accessory genomes. Stars represent the significance of Fisher's exact test for the differential presence of a specific COG category between core and accessory genes. ${ }^{*}$ - $p$-value $<0.05, * *$ - p-value $<5 \times 10^{-5}, * * * \mathrm{p}$-value $<5 \times 10^{-10}$ (FDR-corrected p-values). C) Distribution of COG categories for 760 homologous pairs between SOX and MOX pangenomes, where 51 homologs involve at least one accessory gene (functions listed in Table S7). Ten homologs are accessory in both genomes (incl. two transposases and two restriction-modification system genes). Twenty-eight pairs involve a core gene in MOX and an accessory gene in SOX (incl. 5 genes in the COG category 'Cell wall/membrane/envelope biogenesis', and also transposases, integrases and restriction-modification system genes). Thirteen pairs involve a SOX core and a MOX accessory gene (incl. 3 genes in the COG category 'Coenzyme transport and metabolism').

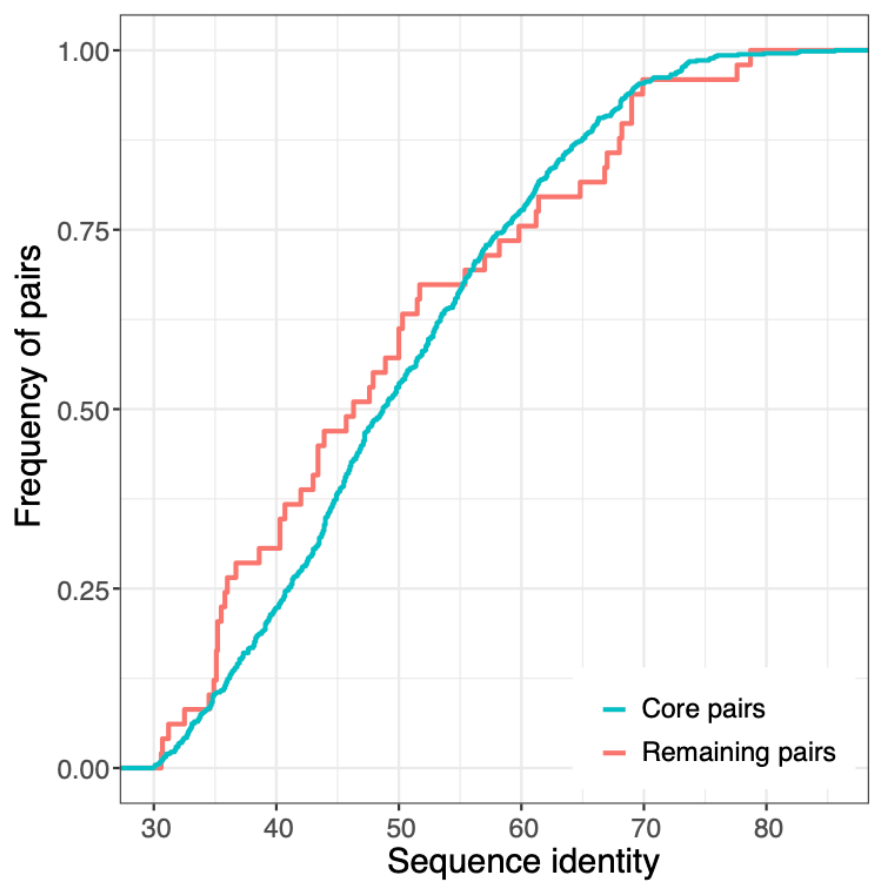

Figure 4. Empirical cumulative distribution of sequence identity between orthologous pairs. Core pairs sequence identity: median 48.7 , maximum 85.6 ; remaining pairs (i.e., containing at least one accessory gene) sequence identity: median 46.3 , maximum 78.7 . 
bioRxiv preprint doi: https://doi.org/10.1101/2021.11.12.468352; this version posted November $13,2021$. The copyright holder for this preprint (which was not certified by peer review) is the author/funder, who has granted bioRxiv a license to display the preprint in perpetuity. It is made available under aCC-BY-NC-ND 4.0 International license.

\section{Tables}

Table 1: Description of SOX and MOX pangenomes.

\begin{tabular}{|c|c|c|c|c|c|c|c|c|c|c|c|c|c|}
\hline & & $\begin{array}{l}\text { Number } \\
\text { of genes }\end{array}$ & $\begin{array}{l}\text { Total } \\
\text { length } \\
\text { (bp) }\end{array}$ & $\begin{array}{l}\text { Median } \\
\text { gene } \\
\text { length }\end{array}$ & $\begin{array}{c}\text { GC } \\
\text { content }\end{array}$ & $\begin{array}{c}\text { Median } \\
\text { (IQR) GC } \\
\text { content } \\
\text { per gene }\end{array}$ & $\begin{array}{l}\text { Number } \\
\text { of SNVs }\end{array}$ & $\begin{array}{l}\text { SNVs/ } \\
\text { kbp }\end{array}$ & $\begin{array}{c}\text { Median } \\
\text { SNVs/kbp } \\
\text { per gene }\end{array}$ & $\begin{array}{c}\text { Number of } \\
\text { genes with } \\
\text { SNVs }\end{array}$ & $\mathrm{pN} / \mathrm{pS}$ & $\begin{array}{c}\text { Median } \\
\text { pN/pS } \\
\text { per gene }\end{array}$ & $\begin{array}{l}\text { Median } \\
\text { pS per } \\
\text { gene }\end{array}$ \\
\hline \multirow[t]{4}{*}{ sox } & Core & 1,522 & $1,386,003$ & 735 & 0.374 & $\begin{array}{c}0.380 \\
(0.0502)\end{array}$ & 17,835 & 12.87 & 11.9 & $1,33288 \%$ & 0.135 & 0.09454 & 0.01613 \\
\hline & $\begin{array}{l}\text { Single-copy } \\
\text { accessory }\end{array}$ & 939 & 863,319 & 480 & 0.356 & $\begin{array}{c}0.331 \\
(0.0808)\end{array}$ & 6,791 & 7.866 & 0 & $46449 \%$ & 0.326 & 0.2432 & 0.009459 \\
\hline & $\begin{array}{l}\text { Multi-copy } \\
\text { accessory }\end{array}$ & 23 & 21,735 & 765 & 0.379 & & 789 & 36.30 & 28.6 & $2296 \%$ & 0.404 & 0.2673 & 0.02461 \\
\hline & Total & 2,484 & $2,271,057$ & & & & & & & & & & \\
\hline \multirow[t]{4}{*}{ MOX } & Core & 2,452 & $1,961,220$ & 637.5 & 0.38 & $\begin{array}{c}0.380 \\
(0.0401)\end{array}$ & 4,585 & 2.338 & 1.63 & $1,63267 \%$ & 0.421 & 0.2656 & 0.003208 \\
\hline & $\begin{array}{l}\text { Single-copy } \\
\text { accessory }\end{array}$ & 379 & 254,967 & 510 & 0.329 & $\begin{array}{c}0.359 \\
(0.0559)\end{array}$ & 245 & 0.9609 & 0 & 81 21\% & 0.514 & 0.2637 & 0.002921 \\
\hline & $\begin{array}{l}\text { Multi-copy } \\
\text { accessory }\end{array}$ & 35 & 19,686 & 402 & 0.377 & & 496 & 25.20 & 17.3 & $3189 \%$ & 0.323 & 0.4145 & 0.01460 \\
\hline & Total & 2,86 & 873 & & & & & & & & & & \\
\hline
\end{tabular}

Median pN/pS and median pS estimated among genes with at least one SNV. bp - base pair, kbp kilobase pair, IQR - interquartile range. 